\title{
Tensile elongation of unidirectional or laminated composites combining a brittle reinforcement with a ductile strain and strain-rate hardening matrix
}

\author{
Amaël Cohades, Andreas Mortensen \\ Laboratory of Mechanical Metallurgy, Institute of Materials, Ecole Polytechnique Fédérale de \\ Lausanne, EPFL Station 12, CH-1015 Lausanne, Switzerland
}

\section{Abstract}

We use the long-wavelength model of Hutchinson and Neale (Hutchinson JW, Neale KW. Acta Metall 1977;25:839) and Ghosh (Ghosh AK. Acta Metall 1977;25:1413) to estimate the uniform tensile elongation of two-phase composites deforming quasistatically according to the equistrain rule of mixtures, in which one phase is ductile while the other fractures progressively according to twoparameter Weibull statistics. We use shear-lag models in the literature to quantify load transfer from the ductile phase to the fractured brittle phase, to estimate the influence of matrix strain and strain-rate hardening, of brittle phase fracture characteristics, and of phase volume and strength ratios, on the composite strain to failure as dictated by the onset of unstable necking. Calculations show that strain and strain-rate hardening of the ductile phase do relatively little to increase the ductility of the composite. Two parameters play a dominant role, namely the brittle phase Weibull modulus and a dimensionless parameter describing load transfer across the two phases. The main practical implication of this analysis is that, to produce reasonably ductile two-phase composites, the best strategy is to aim for small layer thicknesses.

\section{Introduction}

Many composite materials combine a ductile matrix with a brittle reinforcement: ceramic or carbon fibre reinforced metals fall in this category, as do some laminated metal composites and many other layered materials (such as thin film structures or ceramic-coated metals) [1-4]. When such composites are strained in tension the brittle phase develops cracks that cut its fibres or layers in two along a plane normal to the applied load; in laminated composites these are known as "tunnel cracks". Final fracture of the material may then happen in one of two ways. One is sudden brittle failure, caused by a propagative localization of internal damage that cuts abruptly the entire specimen in two; many strongly bonded ceramic or carbon fibre reinforced composites fail in this way [5-7], as do some particle reinforced composites [8]. Alternatively, internal damage accumulates stochastically throughout the material, in gradual and uncorrelated fashion. The composite is then likely to fail by strain localization, reaching its ultimate tensile strength at a smooth maximum along its stress-strain curve, and sometimes deforming significantly thereafter, while deformation concentrates in a portion of the sample's gauge length. Weakly bonded fibre reinforced composites (e.g., [5]), ceramic particle reinforced metals (e.g., [8,9]), two-phase metal alloys [10] or laminated metal composites [11] can all fail in this way. Strain hardening and strain-rate hardening then both play an important role: even small increases in a ductile material's strain hardening rate, or in its strain-rate sensitivity, can noticeably increase the tensile elongation when it is governed by the onset of strain localization. 
J.W. Hutchinson and co-workers [12,13], simultaneously with A.K. Ghosh [14], proposed a simplified approach to predict the tensile elongation of hardening strain-rate sensitive tensile bars. The calculation rests on the long-wavelength approximation, which takes the stress to be uniform across any given cross-section of a tensile bar's gauge section. The gauge section, in turn, is assumed to contain two collinear regions, one slightly narrower and shorter than the other, main, portion of the bar's gauge section. Equating the load carried by either section and integrating their collective deformation numerically taking the main portion to deform at a steady rate, a tensile test conducted on a bar containing a slight imperfection can be simulated. One finds [12-14] that the narrower region starts at some point to deform much faster than the remainder of the bar, indicating that it will soon fail; this gives a prediction of its final tensile elongation. With this model the collective influences of both work hardening and of strain-rate hardening can be accounted for, and the analysis confirms and approximately quantifies the strong role played by strain-rate hardening in delaying the tensile failure of ductile metals and alloys.

The Hutchinson-Neale-Ghosh long-wavelength approximation model was recently extended to derive the uniaxial tensile deformation of equistrain composites (i.e., laminates stressed in their lamination plane or fibre composites stressed along their axis) made of two work hardening and strain-rate sensitive ductile phases [15]. Here, we consider the complementary problem of equistrain composites, also failing in ductile fashion (i.e., with no localized catastrophic propagation of internal damage), in which only one phase is ductile, while the other is a brittle elastic phase that fractures according to Weibull statistics. We again consider the most basic and simple case, namely a strongly bonded unidirectional or laminated composite, which deforms slowly along the fibre or laminate direction. We describe load-sharing near a crack in the brittle phase using classical shear-lag theory, known to apply with good accuracy in fibre reinforced composites [16,17]. More specifically, we assume that load is transferred from the ductile phase back to broken elements of the brittle phase via an interfacial shear stress that equals the instantaneous average flow stress of the ductile phase in shear. This differentiates the present model from global load sharing models that have been proposed so far in the literature for brittle/ductile phase composites [16-19] because here the intact, ductile, phase hardens as it flows. Both the load it carries and the load it transmits to the brittle phase, wherever the latter has fractured, therefore increase as the composite deforms: we examine here how this influences ductile failure of the composite (or, in the parlance of composite mechanics, its failure by global load sharing [16,17]). As will be seen, one finds that despite the many parameters needed to describe this two-phase system, in the end only two dimensionless parameters influence significantly its strain to failure. The reason for this is that the destabilizing effect of internal damage nearly always overrides the stabilizing effect of matrix hardening on the strain to failure of the deforming composite. As a result, strategies for the microstructural optimization of such composites toward increased ductility are relatively simple to formulate, as we show at the end of this contribution after presenting the model and what it predicts.

\section{Governing equations}

\subsection{Geometry and force balance}

Consider a laminated or unidirectional composite made of two components: A (a ductile phase) and B (a brittle phase). The two are strongly bonded and resist debonding as the composite deforms. Along the plane of lamination or along the fibre direction, the uniaxial flow stress can reasonably be assumed to obey the equistrain rule-of-mixtures: 


$$
\sigma_{L M C}=V_{A} \sigma_{A}+V_{B} \sigma_{B}
$$

where $\sigma_{\mathrm{A}}$ and $\sigma_{\mathrm{B}}$ are respectively the average stress in Phase A or and Phase B when each is deformed to the average uniaxial tensile strain of the composite, $\varepsilon$. Parameters $V_{\mathrm{A}}$ and $V_{\mathrm{B}}=1-V_{\mathrm{A}}$ are the volume fractions of Phases $\mathrm{A}$ and $\mathrm{B}$ respectively, and $\sigma_{\mathrm{LMC}}$ is the true (average) stress acting on the composite along the direction of applied stress.

Following Hutchinson and Neale [12], we consider a tensile specimen of the composite, which is assumed to have a uniform cross sectional area exception made for a reduced (also called "nonuniform") portion of cross-sectional area that is only a small fraction $\eta$ thinner and much shorter than the remaining, main ("uniform") part of the specimen. The long-wavelength assumption takes it that the transition to this reduced portion of the tensile bar is sufficiently smooth for the stress to be uniaxial and homogeneous along any cross-section normal to the applied load. The gage section of the tensile bar is thus made of two colinear regions, one slightly wider and much longer than the other. Axial load equilibrium between the uniform and non-uniform portions dictates:

$$
\left[V_{A} \sigma_{A}+V_{B} \sigma_{B}\right] A=\left[V_{A} \sigma_{A, 0}+V_{B} \sigma_{B, 0}\right] A_{0}
$$

where $A$ and $A_{0}$ are the instantaneous cross-sectional area of the reduced and uniform regions respectively. All quantities associated with the uniform region in Eq. (2) and in all what follows are denoted with a subscript 0 . By definition, the initial fractional non-uniformity $\eta$ is:

$$
\eta=\frac{A_{0, i n}-A_{\text {in }}}{A_{0, \text { in }}}
$$

with $A_{\text {in }}$ and $A_{0, \text { in }}$ the initial cross sectional areas of the reduced and uniform portions of the gauge length, respectively.

It can reasonably be assumed that Phase A deforms at constant volume. The true strain of this phase in the reduced $(\varepsilon)$ and uniform $\left(\varepsilon_{0}\right)$ portions of the tensile bar is then related to the cross-sectional areas $\left(A, A_{0}\right)$ or length $\left(L, L_{0}\right)$ of this phase by:

$$
\varepsilon=-\ln \left(\frac{A}{A_{\text {in }}}\right)=\ln \left(\frac{L}{L_{\text {in }}}\right), \quad \varepsilon_{0}=-\ln \left(\frac{A_{0}}{A_{0, \text { in }}}\right)=\ln \left(\frac{L_{0}}{L_{0, \text { in }}}\right)
$$

Phase B on the other hand does not deform at constant volume: it remains elastic and, most importantly, it cracks. Since its lateral (Poisson) contraction is likely to remain small (because its longitudinal elastic strain will not exceed a few percent), we assume hereafter that its cross sectional areas, in both the reduced and uniform sections, remain constant as the composite deforms. As the composite elongates, therefore, force equilibrium between the uniform and reduced sections dictates: 


$$
e^{-\varepsilon} V_{A, 0} \sigma_{A}+V_{B, 0} \sigma_{B}=\frac{1}{1-\eta}\left[e^{-\varepsilon_{0}} V_{A, 0} \sigma_{A, 0}+V_{B, 0} \sigma_{B, 0}\right]
$$

where, again, subscript 0 denotes quantities in the uniform section.

\subsection{Behaviour of the hardening ductile phase}

The ductile phase in the composite is assumed to harden according to a customary constitutional law in which contributions from strain hardening and strain-rate hardening to the flow stress are added [14][15]:

$$
\sigma_{A}=K\left[\varepsilon^{n}+m \ln \left(\frac{\dot{\varepsilon}}{\dot{\varepsilon}_{R}}\right)\right]
$$

here $K$ is the strength constant, $n$ the strain-hardening exponent, $m$ the strain-rate hardening coefficient and $\dot{\varepsilon}_{R}$ a reference strain-rate, typically given the value $5 \cdot 10^{-5} \mathrm{~s}^{-1}[14][15]$.

\subsection{Statistical model for the damaging brittle phase}

The flow stress of the brittle phase (B) is, in the absence of internal damage, linear elastic: $\sigma_{\mathrm{B}}=E \varepsilon$, where $E$ is Young's modulus of Phase B. We assume that as the composite is strained, it sees the gradual and stochastic appearance of cracks that cut the fibre or planar layer of Phase B in two across a plane normal to the tensile axis. Cracking is assumed to follow classical two-parameter Weibull statistics, namely we take it that the cumulative probability of failure for a single brittle phase element (fibre or layer) of length $L$ subjected to uniaxial stress $\sigma$ is [16,20-22]:

$$
P_{B}(\sigma, L)=1-\exp \left[-\frac{L}{L_{0}}\left(\frac{\sigma}{\sigma_{0}}\right)^{\rho}\right]
$$

where $\rho$ is the Weibull modulus, $L_{0}$ is a reference length and $\sigma_{0}$ a reference strength (note that these two parameters, which are often separated for dimensional convenience, are in fact lumped together in the Weibull statistical law and are hence to be considered as a single parameter, $\left.L_{0}\left(\sigma_{0}\right)^{\rho}\right)$.

We assume that the interface remains intact where a crack has cut an element (layer or fibre) of Phase B. Phase Near a crack, Phase A will therefore transmit stress and hence part of the applied load, across the interface, back to Phase B. This causes the average stress within Phase B to build up as one moves along the stress axis away from each crack it contains. The mechanics of this (complex) problem have been the subject of extensive work in composite micromechanics; Refs. [16][17] give detailed in-depth reviews. The simplest ("shear-lag") view of the problem is that wherever the brittle phase has cracked, it is reloaded by the ductile phase via the shear stress that the latter exerts, along the stress direction, across the interface between the two phases. As is customary in dealing with ductile matrix composites (even though this is a strong assumption given the complex stress distributions to be expected with a strain and strain-rate hardening material) we take the interfacial shear stress to be uniform and assume 
that it equals the instantaneous average flow stress in shear of the ductile phase. Adopting the Von Mises criterion and assuming Phase A to be isotropic, we take this to be:

$$
\tau_{s}=\frac{\sigma_{A}}{\sqrt{3}}=\frac{K\left[\varepsilon^{n}+m \ln \left(\frac{\dot{\varepsilon}}{\dot{\varepsilon}_{R}}\right)\right]}{\sqrt{3}}
$$

where, as before, $\varepsilon$ is the local instantaneous composite strain along the direction of tensile loading. This causes a linear build-up of $\sigma_{\mathrm{B}}$ away from each crack that Phase B might contain, Figure 1, up to a distance $\delta$ from the crack, at which $\sigma_{\mathrm{B}}$ has reached the value that would obtain in the absence of a crack, namely $E \varepsilon$. A simple force balance gives [16]:

$$
\delta=\frac{h_{B} \sigma_{B} \sqrt{3}}{2 \sigma_{A}}=\frac{h_{B} E \varepsilon \sqrt{3}}{2 K\left[\varepsilon^{n}+m \ln \left(\frac{\dot{\varepsilon}}{\dot{\varepsilon}_{R}}\right)\right]}
$$

where $\sigma_{\mathrm{B}}=E \varepsilon ; \sigma_{\mathrm{A}}$ is given by Eq. (6) and $h$ is the thickness or the radius of the brittle phase in laminated or fibre composites, respectively. Note that both $\sigma_{\mathrm{B}}$ and $\sigma_{\mathrm{A}}$ evolve as deformation progresses.

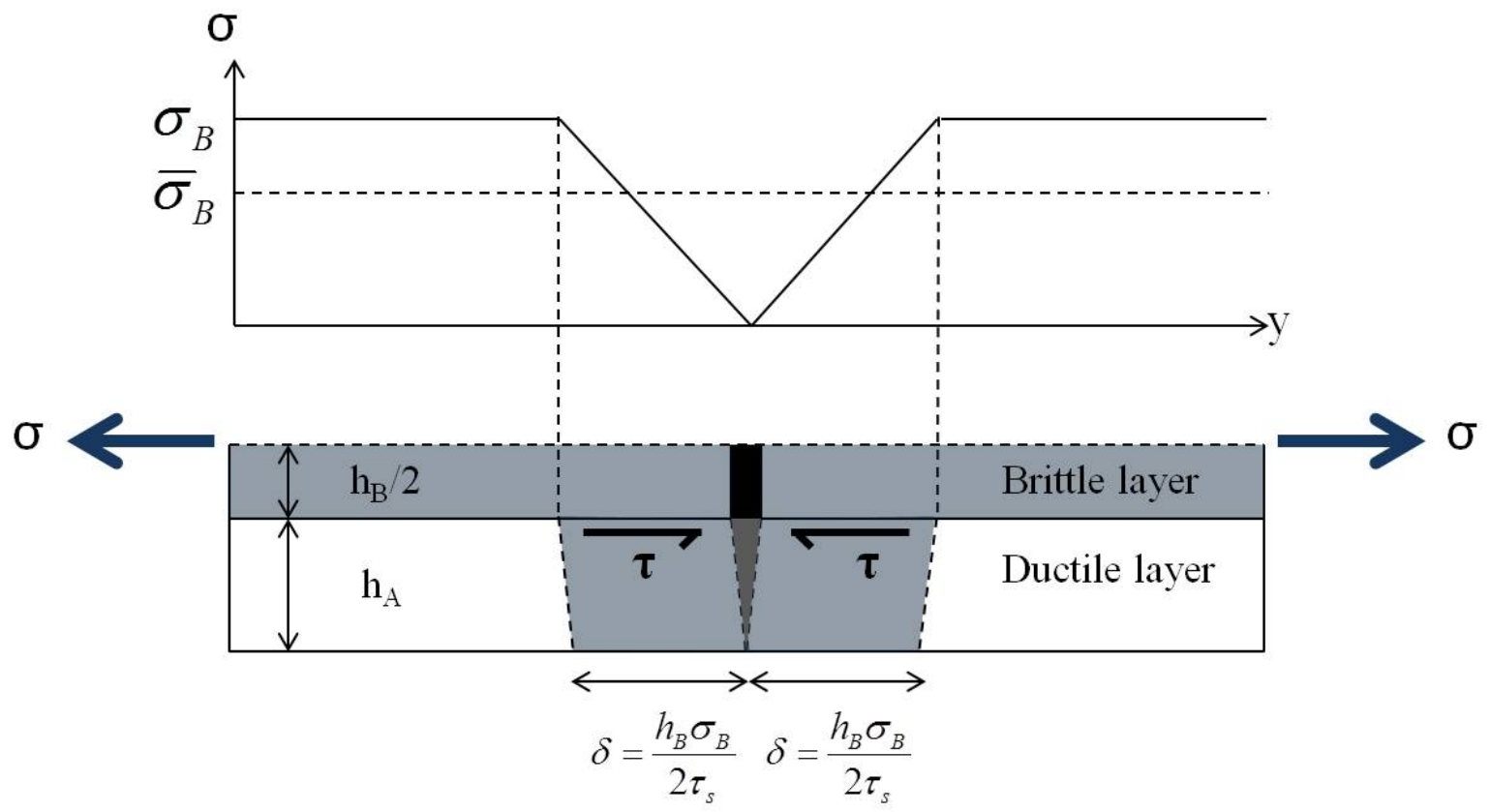

Figure 1: Stress profile around a crack in a brittle layer of the multilayered composite. $\overline{\boldsymbol{\sigma}}_{\boldsymbol{B}}$ is the average stress on the brittle layer, whereas $\sigma_{\mathrm{B}}$ is the remote stress.

The load-bearing capacity of a cracked brittle layer decreases as the number of cracks it contains increases; on the other hand, hardening within the ductile phase decreases $\delta$, which per se increases $\bar{\sigma}_{B}$, raising somewhat the average stress in the brittle phase near the cracks it contains (Figure 1). The average stress in the brittle phase is: 


$$
\bar{\sigma}_{B}=\frac{1}{L} \int_{0}^{L} \sigma_{B}(y) d y
$$

where $\sigma_{B}(y)$ is the stress profile along a statistically representative length $L$ of brittle phase in the composite, measured parallel to the stress axis. It is convenient to rewrite this by summing over all fragments now composing Phase B, sorting contributions by the length $l$ of fragments:

$$
\bar{\sigma}_{B}=\frac{N}{L} \int_{0}^{l} \sigma_{B, l}(y) d y \int_{0}^{\infty} P\left(l, \sigma_{B}\right) d l
$$

where $\sigma_{B, l}$ is the stress profile along a fragment of length $l, N$ is the total number of cracks along length $L$ in Phase B and $P\left(l, \sigma_{\mathrm{B}}\right)$ is given by Eq.(7). With $\tau_{\mathrm{s}}$ the same everywhere at any given instant, one has:

$$
\int_{0}^{l} \sigma_{B, l}(y) d y= \begin{cases}\sigma_{B}\left(1-\frac{\delta}{l}\right) & l \geq 2 \delta \\ \sigma_{B} \frac{l}{4 \delta} & l<2 \delta\end{cases}
$$

Combining Eqs. (11) and (12) gives [16]:

$$
\frac{\bar{\sigma}_{B}}{E \varepsilon}=1-\frac{N}{L} \delta+\frac{N}{L} \int_{0}^{2 \delta}\left[\delta-l+\frac{l^{2}}{4 \delta}\right] P\left(l, \sigma_{B}\right) d l
$$

The second term on the right-hand side accounts for layer fragmentation: it represents the reduced load-bearing capacity of the fragments near their ends. The third term accounts for overlapping of the recovery regions in fragments of length $l<2 \delta$ [16]. In the absence of cracks, the second and third term of Eq. (13) equal zero and the average stress is simply $\sigma_{B}=E \varepsilon$, as should be.

\subsection{Curtin's model}

Curtin $[16,23,24]$ developed an approximate analytical solution to Eq. (13) by considering that, with sufficiently few breaks, the third term can be neglected because fragments of length $l<2 \delta$ are then unlikely. This assumption leads to accurate estimates of the peak load, and hence the tensile strength, of composites in which the fibres carry nearly all the load and the matrix flows at steady stress; unfortunately, as we shall see below, for the present problem Curtin's simplification is not justified. Still, this model has the virtue of leading to simple expressions that expose well the physics of the present problem; we therefore use it first in formulating the problem tackled here.

Curtin's assumption reduces Equation (13) to: 


$$
\frac{\bar{\sigma}_{B}}{E \varepsilon}=\left[1-\frac{1}{2}\left(\frac{E \varepsilon}{\sigma_{R}}\right)^{\rho+1}\right]
$$

where $\sigma_{\mathrm{R}}$ is a reference stress given by:

$$
\sigma_{R}=\left(\frac{L_{0} \cdot \tau_{s} \cdot \sigma_{0}{ }^{\rho}}{h}\right)^{\frac{1}{\rho+1}}
$$

The factor $1 / 2$ in the second term of Eq. (14) expresses the fact that sections of Phase B that carry a reduced load and intersect the composite cross section of interest carry on average one-half of the stress carried by an intact elastic fibre (this comes from the linear load profile of Phase B near cracks) [16]. Curtin showed [16] that Eq. (14) produces a good approximation of the peak stress in composites with a non-hardening matrix for $\rho>1.6$.

Equation (14) suggests the definition of a dimensionless damage parameter:

$$
\omega \equiv\left(\frac{E \varepsilon}{\sigma_{R}}\right)^{\rho+1} \equiv s^{\rho+1}
$$

(note that, in the present problem, $\omega$ varies as the composite deforms because it contains the matrix flow stress). Inserting Eqs. (6), (8) and (15) into Eq. (16) gives:

$$
\omega=\left(\frac{E \varepsilon}{\sigma_{R}}\right)^{\rho+1}=\frac{E^{\rho+1} \cdot h \cdot \sqrt{3}}{L_{0} \cdot \sigma_{0} \rho \cdot K}\left[\frac{\varepsilon^{\rho+1}}{\varepsilon^{n}+m \cdot \ln \left(\frac{\dot{\varepsilon}}{\dot{\varepsilon}_{R}}\right)}\right]
$$

leading to define another dimensionless parameter, which incorporates only fixed physical characteristics of the composite:

$$
\varphi \equiv \frac{E^{\rho+1} \cdot h \cdot \sqrt{3}}{L_{0} \cdot \sigma_{0} \rho \cdot K}
$$

Using Curtin's expression for the average stress in Phase B, Eq. (14), the composite flow stress is now: 


$$
\sigma_{L M C}=V_{A} \cdot K e^{-\varepsilon}\left[\varepsilon^{n}+m \ln \left(\frac{\dot{\varepsilon}}{\dot{\varepsilon}_{R}}\right)\right]+V_{B} \cdot E \cdot \varepsilon\left[1-\frac{1}{2} \varphi\left[\frac{\varepsilon^{\rho+1}}{\varepsilon^{n}+m \cdot \ln \left(\frac{\dot{\varepsilon}}{\dot{\varepsilon}_{R}}\right)}\right]\right]
$$

Combining Eqs. (5) and (19), and defining $\gamma \equiv \frac{V_{A} K}{V_{B} E}$ gives:

$$
\begin{aligned}
e^{-\varepsilon} \cdot \gamma\left[\varepsilon^{n}+m \cdot \ln \left(\frac{\dot{\varepsilon}}{\dot{\varepsilon}_{R}}\right)\right]+\varepsilon\left[1-\frac{1}{2} \varphi\left[\frac{\varepsilon^{\rho+1}}{\varepsilon^{n}+m \cdot \ln \left(\frac{\dot{\varepsilon}}{\dot{\varepsilon}_{R}}\right)}\right]\right] \\
=\frac{1}{1-\eta}\left[e^{-\varepsilon_{0} \cdot \gamma\left[\varepsilon_{0}^{n}+m \cdot \ln \left(\frac{\dot{\varepsilon}_{0}}{\dot{\varepsilon}_{R}}\right)\right]+\varepsilon_{0}}\left[1-\frac{1}{2} \varphi\left[\frac{\varepsilon_{0}^{\rho+1}}{\varepsilon_{0}^{n}+m \cdot \ln \left(\frac{\dot{\varepsilon}_{0}}{\dot{\varepsilon}_{R}}\right)}\right]\right]\right]
\end{aligned}
$$

for the composite in the long-wavelength approximation when the brittle phase average stress is expressed using Curtin's approximation.

Equation (20) gives a relation between the instantaneous values of strain and strain-rate in the uniform and non-uniform sections of the composite tensile bar. When it is solved numerically for $\dot{\varepsilon}$ with $\dot{\varepsilon}_{0}$ fixed it gives values for the strain in both the uniform and reduced sections, versus time. These at first both increase slowly, with the strain in the reduced section only slightly higher than in the remainder of the gauge section. At some point the strain in the reduced section starts deviating to much higher values than in the uniform section: necking has become catastrophically unstable. The strain at that point in the (main) uniform section is taken here as an estimate of the elongation to failure of the material; its value depends somewhat, but only slightly, on the value of $\eta$, provided $\eta$ is only slightly smaller than unity.

By analogy with the behaviour of ductile metals and alloys, the influence of strain-rate hardening in the ductile phase, if any, is expected to occur after the peak stress. At that point, overlapping of the recovery regions near the breaks is likely; Curtin's solution then loses accuracy. Two improvements to Curtin's analysis exist in the literature.

\subsection{Improved models}

Hui et al. [18] derived the exact fragment size distribution $P\left(l, \sigma_{\mathrm{B}}\right)$ and numerically calculated the composite mean stress, thus solving Eq. (13) precisely; however, their solution could not be expressed analytically. An approximate improved expression was given by Neumeister [19], who developed an alternative expression for the average brittle phase flow stress, accounting for the influence of overlapping shear-lag regions. Neumeister's expression is approximate but it fits better Hui et al.'s [18] exact solution than does Eq. (14), particularly at low values of the Weibull exponent $\rho$. 
Both Neumeister's and Hui et al.'s solution can be expressed in terms of the same dimensionless parameter as Curtin's expression, namely $\omega$ defined in Eq. (16). This is seen directly with Neumeister's model [19], which gives the flow stress of the brittle phase as:

$$
\frac{\bar{\sigma}_{B}}{E \varepsilon}=\left[\frac{1}{\omega+1}+\frac{1}{2 \ln (\omega+1)}\left(\frac{\omega}{\omega+1}\right)^{2}\right]
$$

Combining Eqs. (5), (6) and (21) gives for this model the equation to be integrated to derive the composite mean stress and uniform elongation in the long-wavelength approximation.

Hui et al.'s solution [18] expresses the composite mean stress as:

$$
\frac{\bar{\sigma}_{B}}{E \varepsilon}=\frac{1}{s}\left[\int_{0}^{s}\left[\frac{x^{2}}{2}\right] P_{B}(s, x) d x+s \int_{s}^{\infty}\left[(x-s)+\frac{s}{2}\right] P_{B}(s, x) d x\right]
$$

where $P_{B}(s, x)$ is the same probability function as in Eq. (7) and where $s=\frac{E \varepsilon}{\sigma_{R}}$ is the dimensionless stress defined in Eq. (16). Some mathematical transformations have to be applied to Eq. (22) before solving it numerically; these lead to express it as:

$$
\frac{\bar{\sigma}_{B}}{E \varepsilon}=\frac{1}{s}\left[\int_{0}^{s} \exp \left(\frac{-2 Y}{1+\rho}\right) R(Y, \rho) \rho \Psi(Y, \rho) d t\right]
$$

where:

$$
\begin{gathered}
Y=\frac{s^{\rho+1}}{2}=\frac{\omega}{2} \\
R(Y, \rho)=\frac{1-\rho}{\rho}-\frac{Y}{2}+\frac{\left(1-e^{-Y}\right)}{Y} \\
\Psi(Y, \rho)=e^{-2 \Phi(Y)} \\
\Phi(Y)=\frac{\rho}{\rho+1} \int_{0}^{Y} \frac{1-e^{-t}}{t} d t
\end{gathered}
$$

Hui et al. [18] then seek a solution for the composite mean stress having the form: 


$$
\mu(Y)=\frac{2^{\frac{1}{1+\rho}}}{1+\rho} \cdot f(Y) \cdot e^{g(Y)}
$$

where:

$$
\begin{gathered}
f(Y)=Y^{\frac{1}{1+\rho}} \cdot \sum_{k=0}^{\infty} a_{k} Y^{k} \\
g(Y)=-2 Y-2 \frac{\rho}{\rho+1} \sum_{k=0}^{\infty} b_{k} Y^{k}
\end{gathered}
$$

The coefficients $a_{k}$ are given by the following recursive relation:

$$
\begin{gathered}
a_{0}=\frac{1+\rho}{\rho}=a_{1} \\
a_{n+1}=\left[\frac{1+\rho}{(1+\rho)(n+1)+1}\right]\left[\left(-\sum_{k=0}^{n} a_{n-k} c_{k}\right)+\frac{(-1)^{n+1}}{(n+2) !}\right] \text { for } n \geq 1
\end{gathered}
$$

while the $b_{k}$ coefficients are defined as:

$$
b_{k}=\frac{(-1)^{k+1}}{k(k !)}
$$

and finally the $c_{k}$ coefficients are:

$$
c_{0}=-2 \quad c_{k}=-2 \frac{\rho}{\rho+1}(k+1) b_{k+1} \quad \text { for } k \geq 1
$$

As seen, all three models for the average stress in Phase B, including the complete solution by Hui et al. [18], are governed by the same dimensionless parameters, namely the (strain-dependent) parameter $\omega$, which itself contains the dimensionless constant $\varphi$ defined in Eq. (18). Whatever the model used, therefore, the problem is governed by five dimensionless parameters, namely: $n, m, \rho, \gamma$, and $\varphi$. Once these are fixed, one can calculate, in the long-wavelength approximation and with all the assumptions stated above, the uniform elongation as well as the tensile curve of the composite. 
A load balance akin to Eq. (20) is thus written for the model of Hui et al. [18] in the long-wavelength approximation by combining Eqs. (5), (6) and (24) with an evolving shear-stress in the brittle phase as expressed in Eq. (8). As in Ref. [15], we solve numerically this relation using a Taylor-series-based explicit finite difference algorithm, using strain increments $\Delta \varepsilon_{0}=0.0001$, and calculate the value of $\varepsilon$ at each strain increment using the bisection method with an error of $10^{-10}$. Also, for every calculation, $L_{0}$ is set to a standard value of one metre (such that $L_{0}\left(\sigma_{0}\right)^{\rho}$ is expressed in terms of $\sigma_{0}$ only).

An example is given in Figure 2 where the calculated stress-strain curve in the uniform section of a laminated composite combining two steels in equal volumetric proportions is shown. Parameters are from the literature except for $\rho$, which was slightly lowered, for a ductile steel [25-27] and a more brittle steel $[28,29]$. The composite peak stress values differ little between the three models (as already stated by Neumeister [19]) and follow the same trend with increasing $m$ (the Curtin model underestimates the exact solution whereas Neumeister's model overestimates it). On the other hand the three models disagree more significantly past the peak stress; we therefore use hereafter the exact analysis of Hui et al., referring occasionally to Curtin's model when it brings light to the behaviour of the composites considered here.

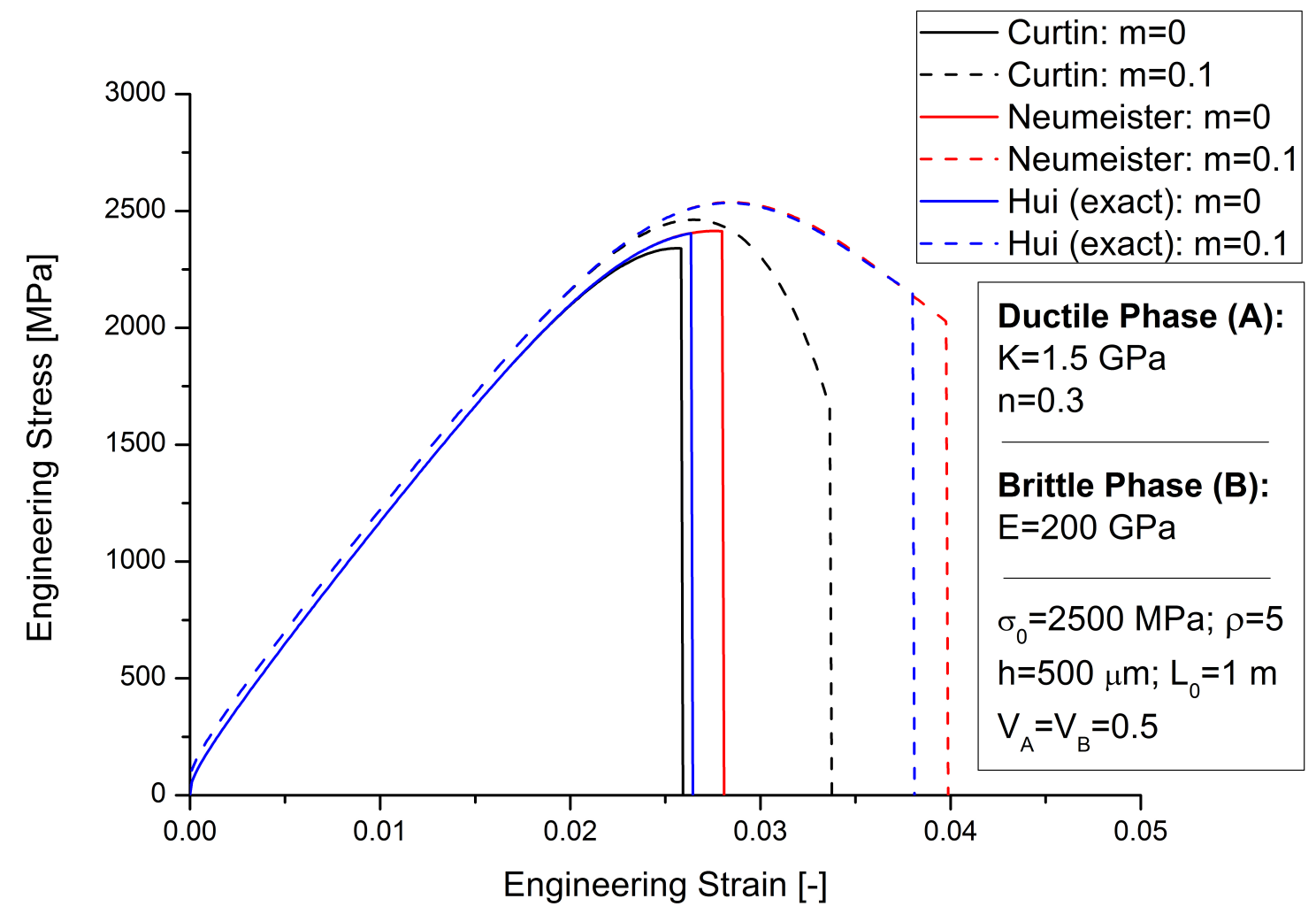

Figure 2: Engineering stress-strain curves in the uniform portion of multilayered composite specimens for Curtin model (Eq. (14)), Neumeister model (Eq. (21)) and Hui model (Eq. (24)). For various values of $m$ and taking $\eta=0.005$.

\section{Results}

Strains in the uniform and non-uniform sections can be obtained by combining the long-wavelength analysis (Eq. (5)) and the Hui model (Eq. (24)). Figure 3 shows what is predicted for the same laminated metal composite as above, combining equal volumes of a ductile steel [25-27] and a 
damaging steel $[28,29]$. Note how strain localizes in the reduced section past a certain strain in the uniform section. The value of strain in the main section $\left(\varepsilon_{0}^{f}\right)$ gives a (conservative) estimate of the composite strain to failure, for two reasons: (i) on average $\varepsilon$ exceeds $\varepsilon_{0}$, and (ii) near the point at which the applied tensile load peaks, the slope of the true stress-true strain curve is also low, such that a small difference in stress between the two regions translates into a much larger difference in strain. Both Figure 2 and Figure 3 show that, as in Ref. [15] and for metals and alloys more generally, a finite strain-rate sensitivity can delay the onset of instability, increasing the tensile elongation that is reached before the neck elongation increases toward infinity; note also how a finite value of $m$ causes a more gradual departure of $\varepsilon$ as compared to $\varepsilon_{0}$. Figure 3 also shows how decreasing the stiffness of the brittle phase, all else constant, can increase the composite strain to failure.

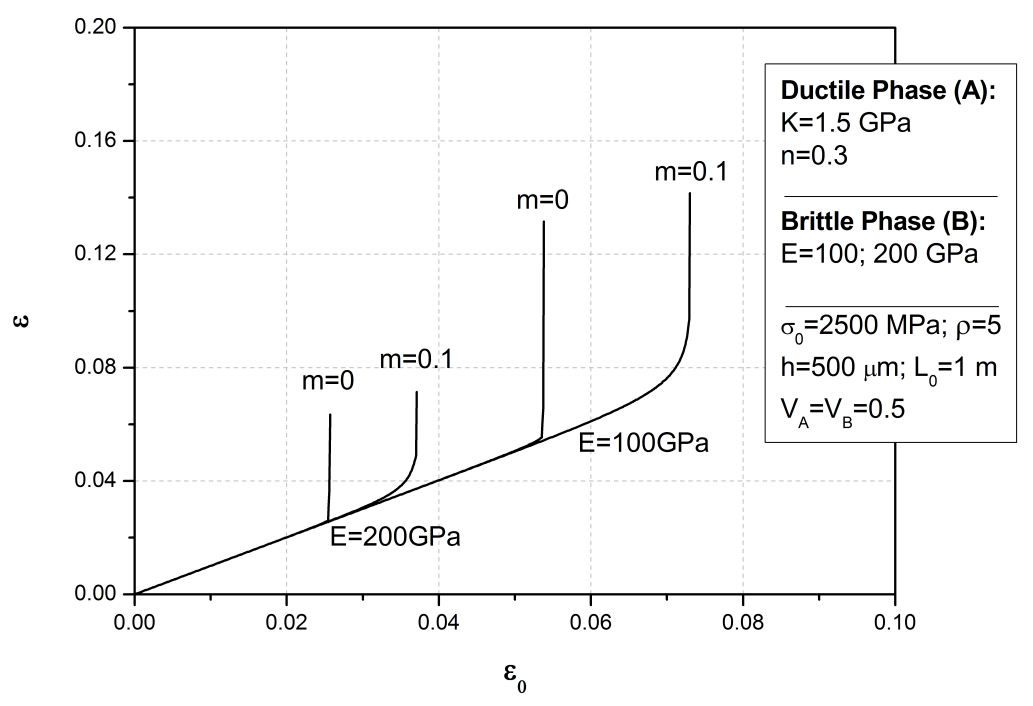

Figure 3: Variation of the strain in the non-uniform section $(\eta=0.005), \varepsilon$, with the strain in the uniform section, $\varepsilon_{0}$, of the LMC (parameter values are given in the insert and on the graph).

Of the five parameters characterizing the model, $\rho$ is expected to be influential since it appears as an exponent of $E$ and $\sigma_{0}$. Figure 4 shows, for the same LMC combining a ductile steel [25-27] with a damaging steel $[28,29]$, the strain to failure plotted versus the Weibull modulus: increasing $\rho$ leads to a decrease of $\varepsilon_{0}^{f}$. This influence appears directly in Curtin's model, Eq. (19): increasing $\rho$ causes a more abrupt decrease in the composite flow stress with increasing strain, which per se tends to destabilize the composite against necking. Figure 4 also gives a first view of the influence exerted by $n$ and $m$ on the composite ductility. An increase in strain-rate hardening exponent $m$ (Figure 4 (a)) gives, at fixed $\rho$, a higher failure strain; however, this influence decreases as $\rho$ increases and is almost negligible when $\rho=10$. On the other hand, an increase in the strain hardening exponent $n$ (Figure 4 (b)) gives, for fixed $\rho$, a lower failure strain. This unexpected influence, which decreases as $\rho$ increases, is caused by the fact that, when the strain hardening exponent $n$ increases keeping all else constant, the reference strength $\sigma_{\mathrm{R}}$ (Eq. (15)) decreases since $\varepsilon<1$. This in turn increases the damage parameter $\omega$ (Eq. (16)) and therefore decreases the uniform strain to failure. The highest influence of strain hardening parameters is observed for $\rho=2$; however, the effect is in practice smaller than that, since most realistic values for brittle metals and engineering ceramics give $\rho$ between 5 and 20 (roughly, $\rho=3-20$ for ceramics, $\rho=8-20$ for powder metallurgy steels, $\rho>30$ for wrought steels) $[20-22,28-36]$. 


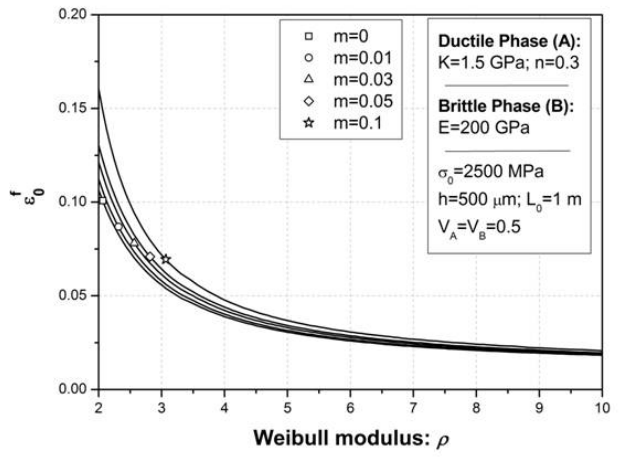

(a)

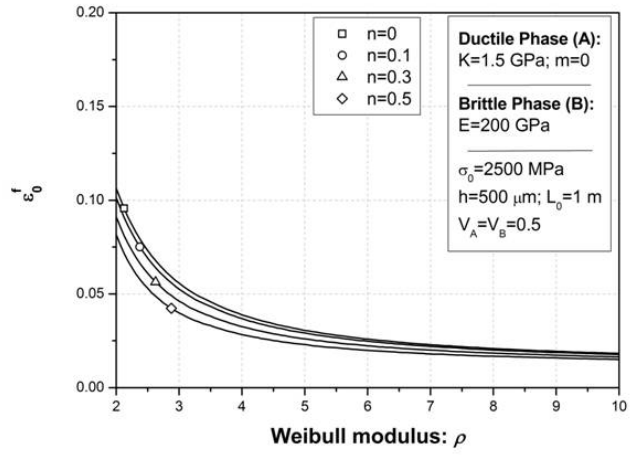

(b)

Figure 4: Maximum attainable strain in the uniform section, $\boldsymbol{\varepsilon}_{\mathbf{0}}^{\boldsymbol{f}}$, of an LMC as a function of the Weibull modulus $\rho$, for various parameters (see insert) and $\eta=0.005$. (a) varying $m$; (b) varying $n$.

We now examine more systematically the influence exerted by the five dimensionless parameters governing the problem: $n, m, \rho, \gamma$ and $\varphi$, taking $n$ between 0 and 0.5 and $m$ between 0 and 0.1 , the Weibull modulus $\rho$ between 5 and 10 (above 10 the ductile phase influence becomes negligible), and $\gamma$ between 0 and 1 . These ranges cause the dimensionless parameter $\varphi$ to be spread over a relatively wide scale, see Table 1 (note that some parameter combinations are not physically realistic, e.g., high $\sigma_{0}$ with low $E$ or vice-versa) [16,28-35,37-45].

Table 1: Typical $\varphi$ values as a function of $\rho, h, \sigma_{0}, K$ and $E$.

\begin{tabular}{|c|c|c|c|c|c|c|c|c|c|c|c|}
\hline \multirow{2}{*}{\multicolumn{2}{|c|}{$\rho=5$}} & 100 & 100 & 100 & 5000 & 5000 & 5000 & 10000 & 10000 & 10000 & $\sigma_{0}[\mathrm{MPa}]$ \\
\hline & & 50 & 200 & 500 & 50 & 200 & 500 & 50 & 200 & 500 & $\mathrm{E}[\mathrm{MPa}]$ \\
\hline 0.001 & 100 & $3 . E+13$ & 1. $E+17$ & $3 . E+19$ & $9 . \mathrm{E}+04$ & 4.E+08 & $9 . E+10$ & $3 . \mathrm{E}+03$ & 1. $\mathrm{E}+07$ & $3 . E+09$ & \\
\hline 0.001 & 5000 & $5 . E+11$ & $2 . E+15$ & $5 . E+17$ & $2 . E+03$ & 7.E+06 & 2.E+09 & $5 . \mathrm{E}+01$ & 2. $\mathrm{E}+05$ & $5 . \mathrm{E}+07$ & \\
\hline 0.000001 & 100 & $3 . E+10$ & 1. $E+14$ & $3 . E+16$ & $9 . \mathrm{E}+01$ & $4 . E+05$ & $9 . \mathrm{E}+07$ & $3 . \mathrm{E}+00$ & 1.E+04 & $3 . E+06$ & \\
\hline 0.000001 & 5000 & $5 . E+08$ & 2. $E+12$ & $5 . E+14$ & $2 . E+00$ & 7.E+03 & $2 . E+06$ & 5.E-02 & 2. $\mathrm{E}+02$ & $5 . \mathrm{E}+04$ & \\
\hline $\mathrm{h}[\mathrm{m}]$ & $\mathrm{K}[\mathrm{MPa}]$ & & & & & & & & & & \\
\hline \multirow{2}{*}{\multicolumn{2}{|c|}{$\rho=7$}} & 100 & 100 & 100 & 5000 & 5000 & 5000 & 10000 & 10000 & 10000 & $\sigma_{0}[\mathrm{MPa}]$ \\
\hline & & 50 & 200 & 500 & 50 & 200 & 500 & 50 & 200 & 500 & $\mathrm{E}[\mathrm{MPa}]$ \\
\hline 0.001 & 100 & 7.E+18 & 4. $E+23$ & 7.E+26 & $9 . \mathrm{E}+06$ & $6 . E+11$ & $9 . E+14$ & 7.E+04 & 4.E+09 & 7.E+12 & \\
\hline 0.001 & 5000 & $1 . E+17$ & 9. $\mathrm{E}+21$ & 1.E+25 & 2.E+05 & 1.E+10 & $2 . E+13$ & 1. $\mathrm{E}+03$ & 9. $\mathrm{E}+07$ & $1 . \mathrm{E}+11$ & \\
\hline 0.000001 & 100 & 7.E+15 & 4. $E+20$ & 7.E+23 & $9 . E+03$ & $6 . \mathrm{E}+08$ & $9 . E+11$ & 7.E+01 & 4. $E+06$ & 7.E+09 & \\
\hline 0.000001 & 5000 & 1.E+14 & 9. $E+18$ & $1 . E+22$ & $2 . E+02$ & $1 . E+07$ & 2.E+10 & 1. $\mathrm{E}+00$ & 9. $\mathrm{E}+04$ & 1.E+08 & \\
\hline $\mathrm{h}[\mathrm{m}]$ & $\mathrm{K}[\mathrm{MPa}]$ & & & & & & & & & & \\
\hline \multirow{2}{*}{\multicolumn{2}{|c|}{$\rho=10$}} & 100 & 100 & 100 & 5000 & 5000 & 5000 & 10000 & 10000 & 10000 & $\sigma_{0}[\mathrm{MPa}]$ \\
\hline & & 50 & 200 & 500 & 50 & 200 & 500 & 50 & 200 & 500 & $\mathrm{E}[\mathrm{MPa}]$ \\
\hline 0.001 & 100 & $8 . E+26$ & 4. $E+33$ & 8.E+37 & 9. $\mathrm{E}+09$ & 4.E+16 & $9 . \mathrm{E}+20$ & 8.E+06 & $4 . E+13$ & $8 . E+17$ & \\
\hline 0.001 & 5000 & $2 . \mathrm{E}+25$ & 7. $\mathrm{E}+31$ & 2. $\mathrm{E}+36$ & 2. $\mathrm{E}+08$ & 7.E+14 & 2.E+19 & 2. $\mathrm{E}+05$ & 7.E+11 & $2 . \mathrm{E}+16$ & \\
\hline 0.000001 & 100 & $8 . E+23$ & 4. $\mathrm{E}+30$ & 8.E+34 & 9. E+06 & $4 . \mathrm{E}+13$ & 9. E+17 & 8. $\mathrm{E}+03$ & 4. $\mathrm{E}+10$ & 8.E+14 & \\
\hline 0.000001 & 5000 & $2 . \mathrm{E}+22$ & 7.E+28 & 2.E+33 & 2. $\mathrm{E}+05$ & 7.E+11 & 2.E+16 & 2. $\mathrm{E}+02$ & 7. $\mathrm{E}+08$ & $2 . E+13$ & \\
\hline $\mathrm{h}[\mathrm{m}]$ & $\mathrm{K}[\mathrm{MPa}]$ & & & & & & & & & & \\
\hline
\end{tabular}




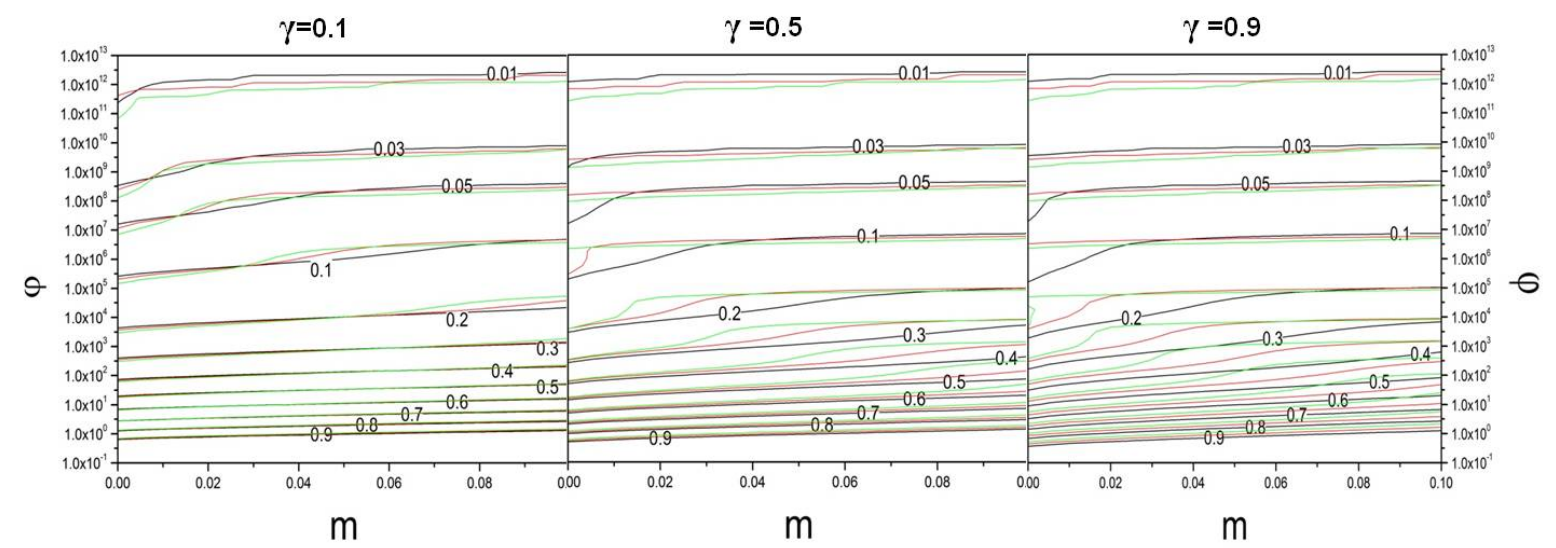

(a)

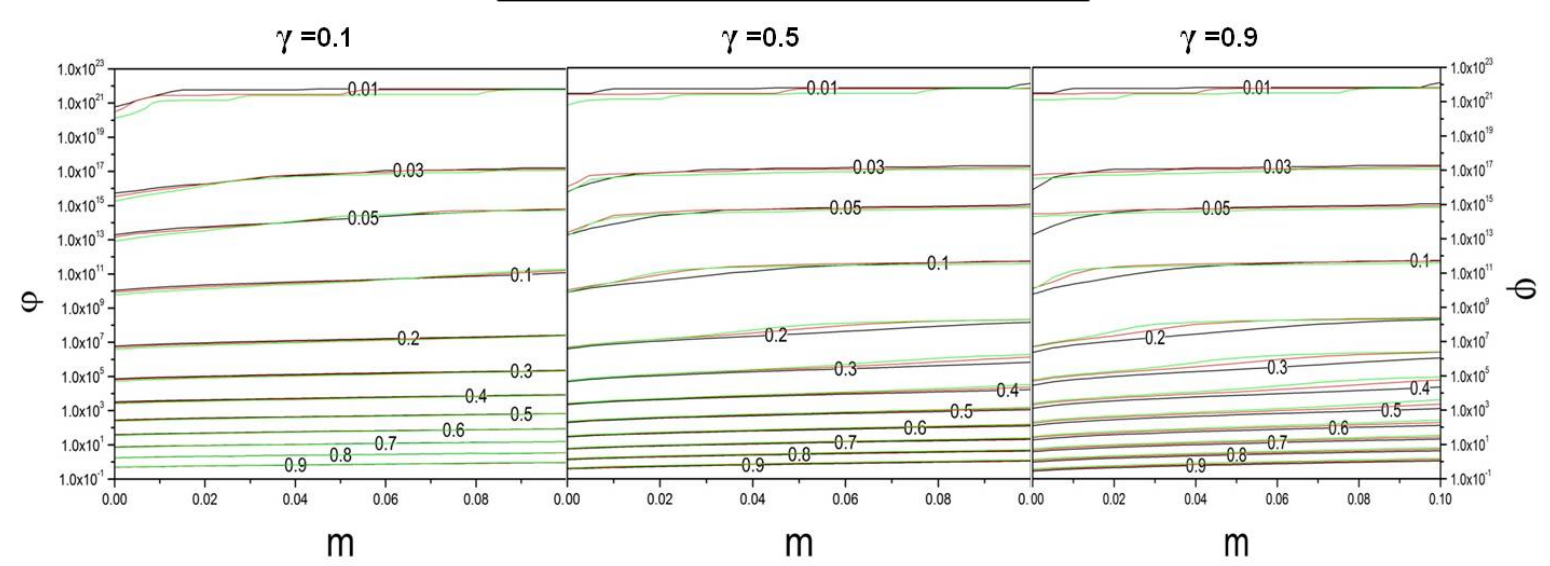

(b)

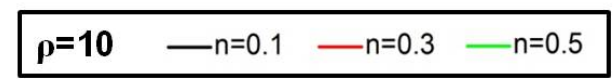

Figure 5: Lines of constant predicted uniform strain to failure $\left(\varepsilon_{\mathbf{0}}^{f}\right)$ of the composite plotted versus dimensionless parameter $\varphi$ and the strain-rate hardening exponent $m$ for three values of $\gamma(\gamma=0.001,0.01$ and 0.1$)$. (a) $\rho=5$; (b) $\rho=10$.

One can see in Figure 5 a few jumps in the predicted ductility around a small range of variation of either $m, n$, or $\gamma$. Its origin is hinted at in Figure 3: note how the transition to instability is abrupt when $m=0$, and more gradual at $m=0.1$. Figure 6 shows that a similar jump occurs, all else constant, as $\gamma$ increases. This figure additionally shows that predictions are relatively insensitive to the time step used in integration: curves calculated with $10^{4}$ or $10^{5}$ iterations are similar, suggesting that the jump is not a numerical artefact. Figure 6 adds to Figure 7 predictions for a wider range of $\gamma$ values (with the integration step now constant), and superimposes on the plot the (dimensionless) load borne by each of the two phases in the reduced portion of the gauge length, with values given on the horizontal axis versus the strain $\varepsilon$ in that region, on the vertical axis of the plot. Note how, when the load-bearing capacity of the ductile phase is very low ( $\gamma=0.0005$ and 0.005 , the black and red curves), tensile instability is triggered when the brittle phase reaches its peak stress. There is then essentially no influence exerted by the ductile phase; this is as one would expect. As the load-bearing capacity of the ductile phase (or in other words, as $\gamma$ ) increases, there is a shift in the point where tensile instability is reached, to a (slightly $\gamma$-dependent) point situated a little after the inflection point at which the (negative) derivative of the brittle phase stress-strain curve reaches a minimum. What this suggests is that with $m=0$, the observed jump in the strain to failure of the composite is linked with the inflection point in the brittle phase flow curve. If the composite survives up to this point, the fact that the brittle 
phase work hardening rate starts to increase gives the composite a temporary lease on resistance to necking. This is then lost when the ductile phase rate of work hardening has decreased sufficiently. Figure 7 (b) shows the effect of a change in $\varphi$, all else constant: the same transitions are found, at corresponding points. Additionally, the graph shows explicitly how increasing the value of $\varphi$, which governs damage in the brittle phase, decreases the composite failure strain and also decreases the amplitude of the transition behaviour (as is visible in Figure 5). The role played by the inflection point in the brittle phase flow stress is also corroborated by the fact that such jumps in strain to failure are not observed when Curtin's equation, which predicts no inflection point in the brittle phase stressstrain curve, is used instead of the full solution by Hui et al. (Eq. (14)). The inflection point in the brittle phase average stress is caused in the model of Hui et al. by the fact that, past a certain strain, overlapping recovery regions in the brittle phase are reloaded again through the increased ductile phase flow stress: the mechanics of flow stabilization by the ductile phase are thus a complex result of its dual roles, in load-bearing and in load-transfer.

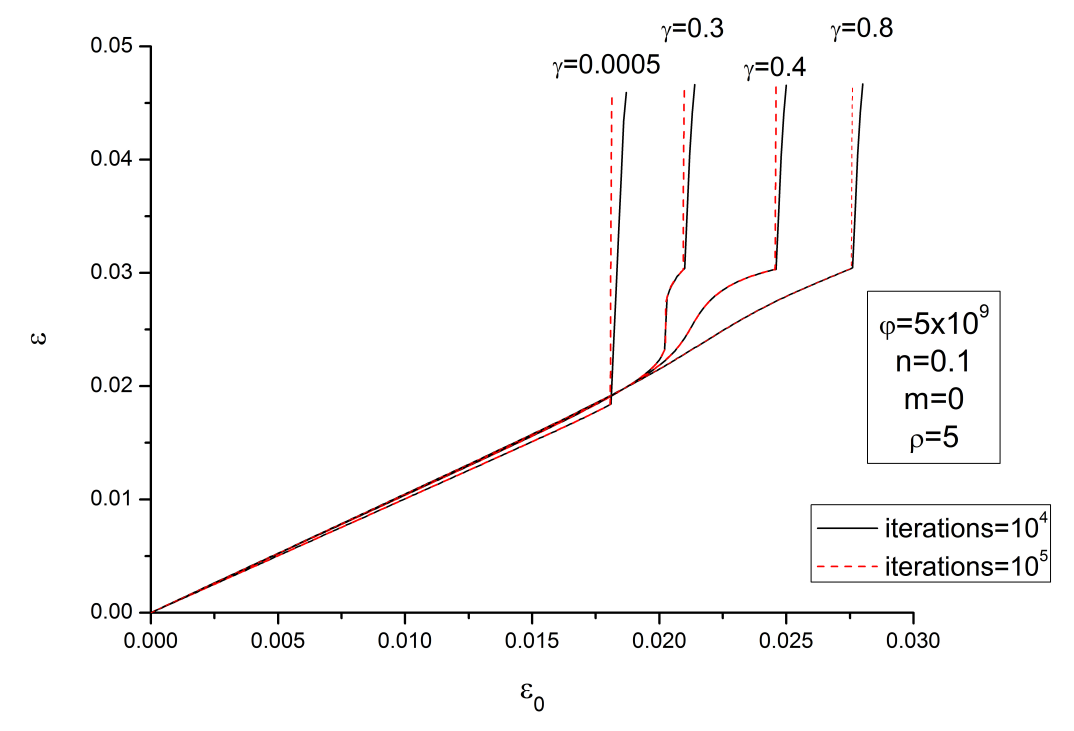

Figure 6: Strain in the non-uniform section, $\varepsilon$, as a function of the strain in the uniform section, $\varepsilon_{0}$, for different values of $\gamma$, two iteration numbers and constant values of $\varphi, n, m$ and $\rho$. 


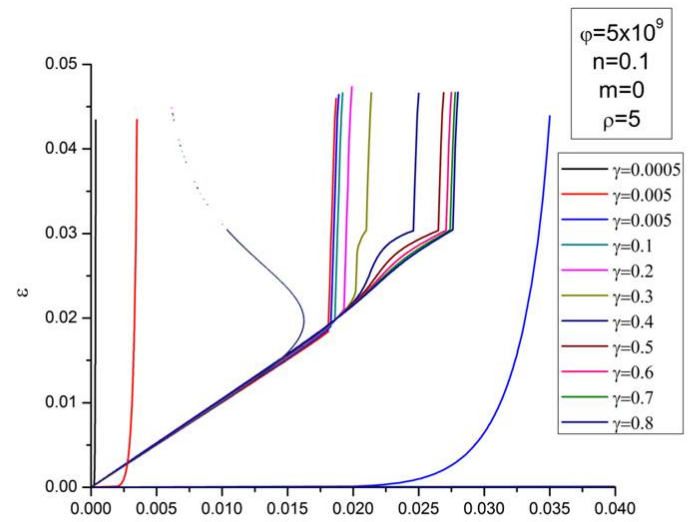

(a) $\varepsilon_{0} ; e^{-\varepsilon} \cdot \gamma\left[\varepsilon^{n}+m \ln \left(\frac{\dot{\varepsilon}}{\dot{\dot{\varepsilon}_{R}}}\right)\right] ; \frac{\sigma_{B}}{V_{B} \cdot E}$

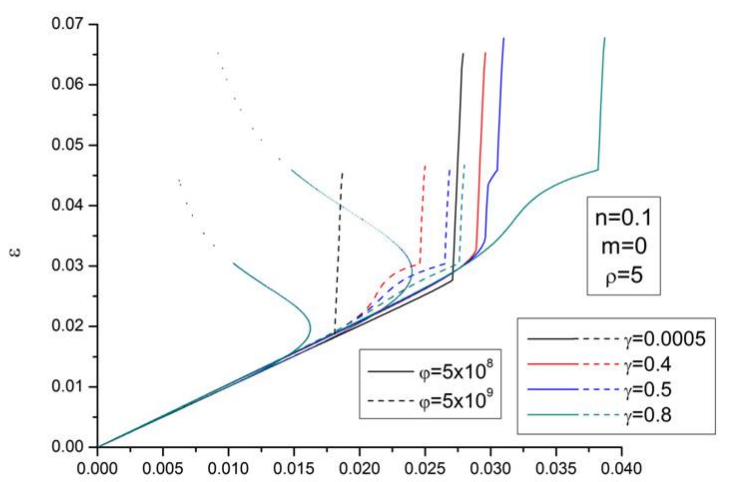

(b)

Figure 7: (a): Strain in the non-uniform section, $\varepsilon$, versus: (i) the strain in the uniform section, $\varepsilon_{0}$ (ii) the dimensionless load borne by the ductile phase (only $\gamma=0.0005,0.005,0.05$ are shown on the graph, other curves being off-range) (iii) the dimensionless load borne by the brittle phase (curves for all $\gamma$ values superimpose), for different values of $\gamma$ and constant marked values of $\varphi, n, m$ and $\rho$. (b): Strain in the non-uniform section, $\varepsilon$, versus: (i) the strain in the uniform section, $\varepsilon_{0}$ (ii) the dimensionless load borne by the brittle phase (curves for all $\gamma$ values superimpose), for two values of $\varphi$, four values of $\gamma$ and constant values of $n, m$ and $\rho$ (indicated on the graph).

Figure 8 shows what happens to predictions in Figure 6 when the ductile phase also exhibits strain-rate hardening $(m=0.1)$. Trends remain the same: $\varepsilon$ deviates overall in the same way from $\varepsilon_{0}$, and also the ductile phase has the same (limited) effect on the elongation to failure. What is new is the presence of a step in the ductile phase flow stress; this is caused by strain-rate hardening, which raises the ductile phase flow stress, in turn raising the average stress in the brittle phase, once deformation in the reduced section starts accelerating. This in turn delays the instability, rounding the $\varepsilon$ versus $\varepsilon_{0}$ curve near the transition to failure: there lies the mechanism whereby strain-rate hardening increases the strain to failure of the composite. As a result, the brittle phase flow stress now depends somewhat on $\gamma$ past its peak. The reason is that $\gamma$ affects the level of ductile phase strain-rate hardening once neck deformation accelerates, causing $\gamma$ to influence $\delta$ (Eq. 9) and therefore the damage parameter $\omega$ (Eq. 17), affecting in turn the flow stress in Phase B. 


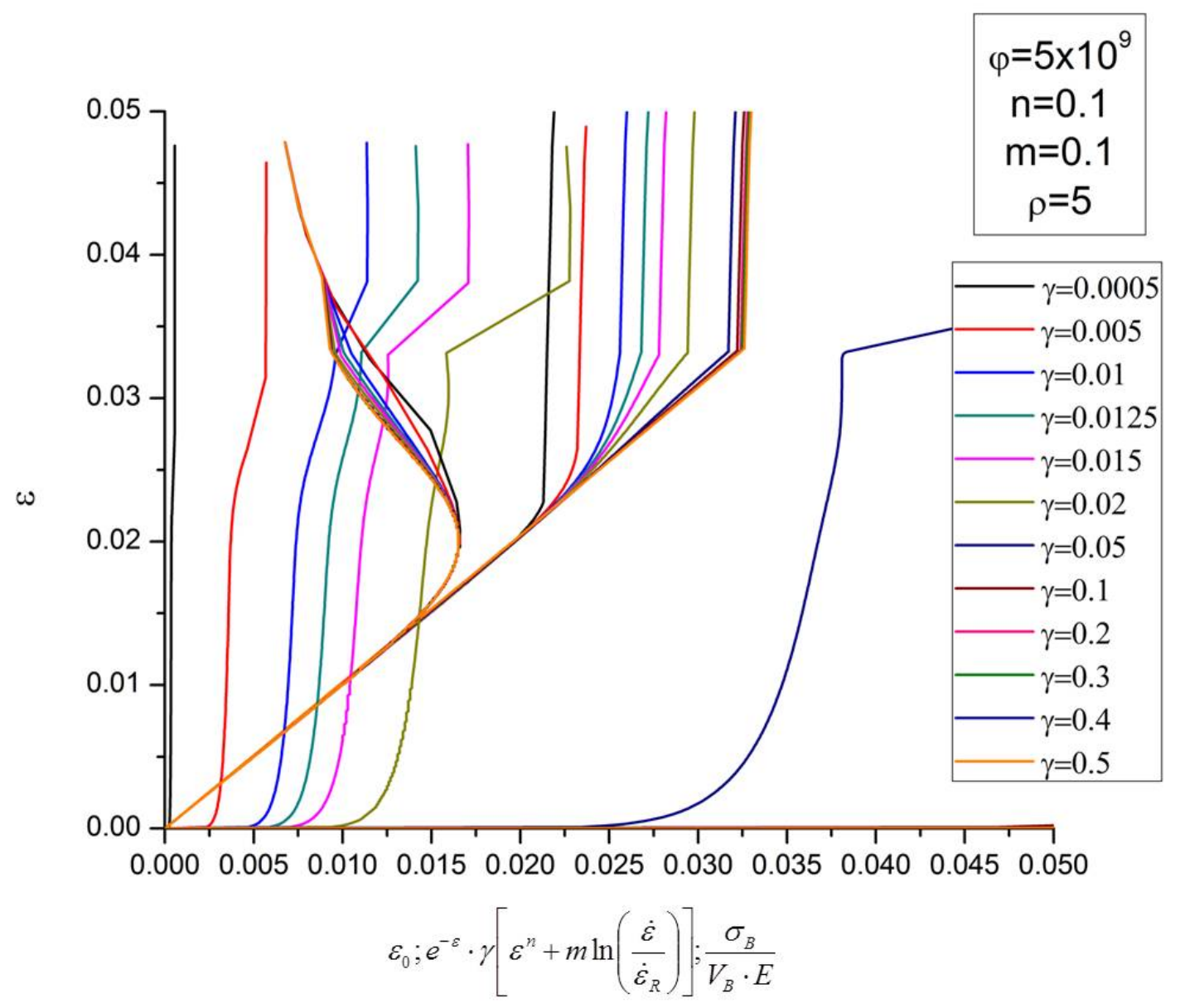

Figure 8: Strain in the non-uniform section, $\varepsilon$, versus: (i) the strain in the uniform section, $\varepsilon_{0}$ (ii) the dimensionless load borne by the ductile phase (only curves for value of $\gamma$ up to 0.05 are shown on the graph, other curves being off-range) (iii) the dimensionless load borne by the brittle phase, for different values of $\gamma$ and constant marked values of $\varphi, n, m$ and $\rho$.

Figure 9 shows the influence of $\rho$ using maps of the strain to failure versus $\varphi$ and $\gamma$ for $\rho=5,7$ or 10 with hardening parameters of the ductile phase set to $n=0.5$ and $m=0.1$. These graphs confirm the minor role played by $\gamma$ : the strain to failure of the composite is mostly dictated by $\varphi$ and $\rho$. Increased $\rho$ gives higher elongations at fixed $\varphi$; this is explained by the fact that increasing $\rho$ while keeping $\varphi$ constant leads to a lower value of damage parameter (Eq. (17), keeping in mind that $\varepsilon$ will nearly always stay below unity). It is worth noting that if one keeps all dimensional physical system parameters fixed and varies only the Weibull exponent $\rho$, its influence will be different since its variations will then also affect $\varphi$ (Eq. 18); this will be detailed below. 
(a)

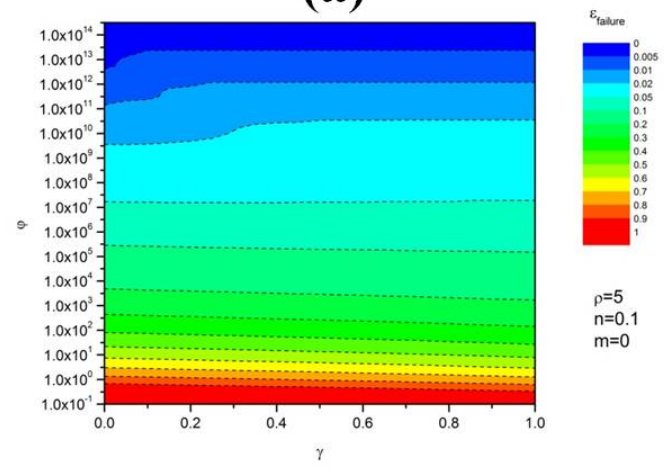

(b)

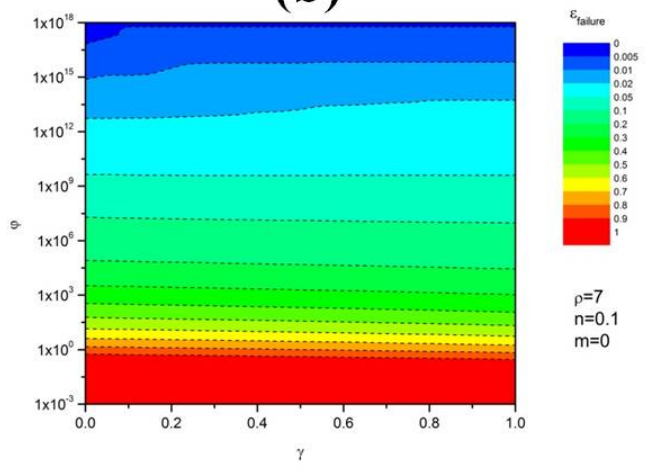

(c)

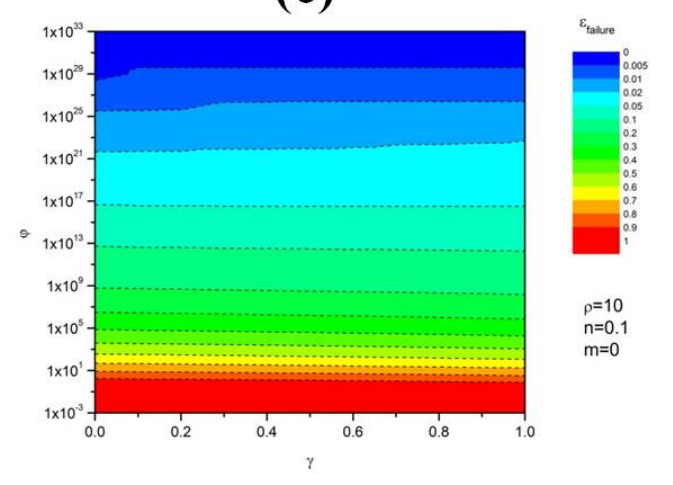

Figure 9: Maximum attainable strain in the uniform section of a composite $\left(\varepsilon_{0}^{f}\right)$ made of a brittle phase and a ductile phase, as a function of the brittle phase dimensionless parameter $\varphi$ (Eq. (18)) and the load sharing fraction $\gamma$, with ductile phase parameters $n=0.5$ and $m=0.1$, and for $\eta=0.005$ : (a) $\rho=5$; (b) $\rho=7$ (c) $\rho=10$.

\section{Engineering implications}

From a physical standpoint, what transpires is that the uniform elongation of ductile/brittle phase composites, as dictated by tensile instability and within the framework of the present analysis, is chiefly governed by properties of the damaging brittle phase. Strain and strain-rate hardening in the ductile phase are of some, but only limited, assistance in delaying failure by the onset of unstable necking in such materials. All relevant dimensionless parameters but $\varphi$ (Eq. 18) and the Weibull modulus $\rho$ have only a minor influence on the composite elongation to failure. Load-bearing by the ductile phase, and the stress it transmits to the brittle phase where the latter has cracked, buy the material at most a small lease of life against the necking instability if one measures ductility by the elongation of the main section of the tensile bar, as we have done here.

We therefore now draw a map that gives, in coordinates of $\varphi$ and $\rho$ and with adequate precision given the weak influence of all other parameters, the composite uniform elongation to failure. This is shown in Figure 10, which plots iso-deformation lines for three combinations of $n$ and $m$ covering a wide range in their possible values, keeping $\gamma$ set to zero (thus ignoring the role of load-bearing by the matrix since this will not increase much the composite elongation, Figure 9; this leads predictions to be slight underestimations). As seen, in composites deforming to high elongation (low values of $\varphi$ ), it is mainly $\varphi$ that governs the elongation. Where $\rho$ plays (at fixed $\varphi$ ) a more important role is where elongations are low: there, its influence can be considerable, as shown by the high slope of the iso- 
deformation lines or by the increase in tensile elongation that is, for example, brought by going from $\rho$ $=7$ to $\rho=15$ when $\varphi$ equals $10^{12}$ : the ductility then goes roughly from 1 to $10 \%$.

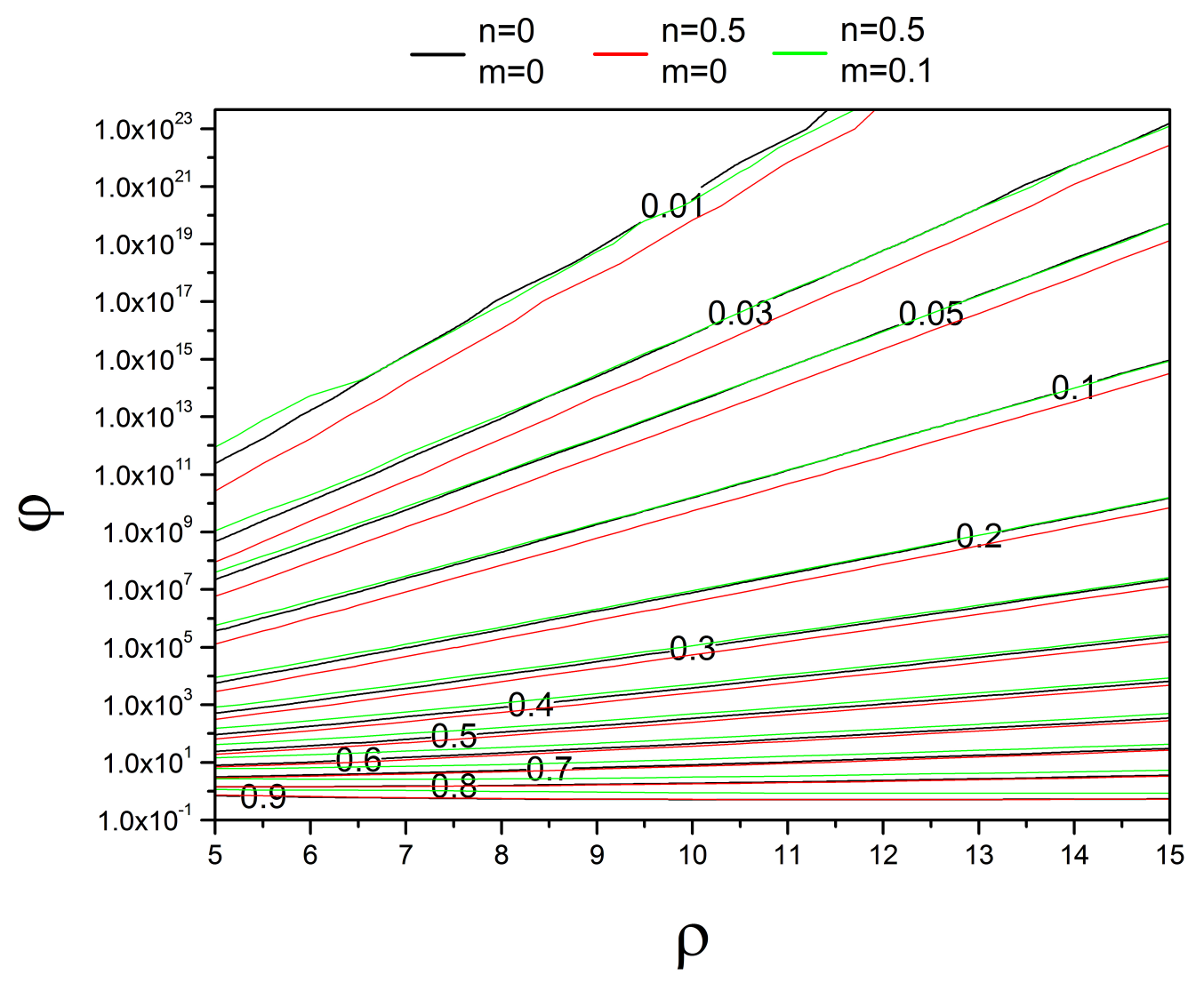

Figure 10: Map of the predicted uniform tensile elongation to failure $\left(\varepsilon_{\mathbf{0}}^{f}\right)$ as a function of the dimensionless parameter $\varphi$ and the Weibull modulus $\rho$, for $\eta=0.005$ and $\gamma=0$. Iso-deformation lines give the uniform strain to failure, for three ductile phase parameters combinations: $n=m=0 ; n=0.5 \& m=0 ; n=0.5 \& m=0.1$.

Table 2 gives relevant physical properties of a few brittle metals in which fracture statistics have been reported in the form of two-parameter Weibull statistics (often, three-parameter Weibull statistics are given instead [31,32,35,37,46-54]); note that these are often for deformation at temperatures well below ambient. Let us consider a "typical" medium-strength brittle steel, with $E=210 \mathrm{GPa}, \sigma_{0}=$ 1,500 MPa and $L_{0}=1 \mathrm{~m}$, leaving the Weibull parameter $\rho$ as a free-floating variable. Let us pair it with alloy $6061(K=550 \mathrm{MPa}, n=0.11, m=0$ [55]).

Returning to the definition of $\varphi$ (Eq. (18)) we write:

$$
\log (\varphi)=\rho \log \left(\frac{E}{\sigma_{0}}\right)+\log \left(\frac{E \cdot h \cdot \sqrt{3}}{L_{0} \cdot K}\right)
$$

This defines a line of slope 2.15 and intercept (-1.48) that can be drawn directly over the map of predicted elongations, as shown in Figure 11 (here $n$ and $m$ have now been fixed at 0.3 and 0 , respectively). As seen, it turns out that the line comes close to that giving material combinations that have a uniform elongation to failure of only $1 \%$ : this is thus the predicted elongation to failure no 
matter what the value of the Weibull parameter $\rho$ might be with this system. To improve the elongation of the laminate, two options are available: (i) decreasing the slope of this line if $\rho$ is high, meaning, for a given Phase A and hence with $E$ roughly constant, increasing its strength as measured by $\sigma_{0}$, or (ii) decreasing the second term between parentheses on the right-hand side of Eq. (25), which means in practice either decreasing $h$ or increasing $K$. There is, for a given material combination, limited flexibility with regard to strength constants $\left(\sigma_{0}\right.$ or $K$ are not easily changed by several orders of magnitude); therefore the main parameter that can move the line drawn by Eq. (25) down significantly is the laminate thickness, $h$. As a bonus, once $h$ falls near or below $10 \mu \mathrm{m}$, this will generally also increase $K$ (Refs. [56,57] give reviews of dislocational layer hardening mechanisms in small scale composites). In the present example, decreasing $h$ from $50 \mu \mathrm{m}$ to $50 \mathrm{~nm}$ will shift the line down, into a range where elongations of several percent can now be expected, particularly if $\rho$ is low, Figure 11. Numbers will change from one system to another (e.g., $\log \left(\frac{E}{\sigma_{0}}\right)$ varies from 1.3 to 3.8 across systems listed in Table 2); however, the line of reasoning that precedes and its main conclusion, remain. The most robust and potent strategy in designing laminated composites having some stability against necking and hence reasonable ductility is to reduce $h$.

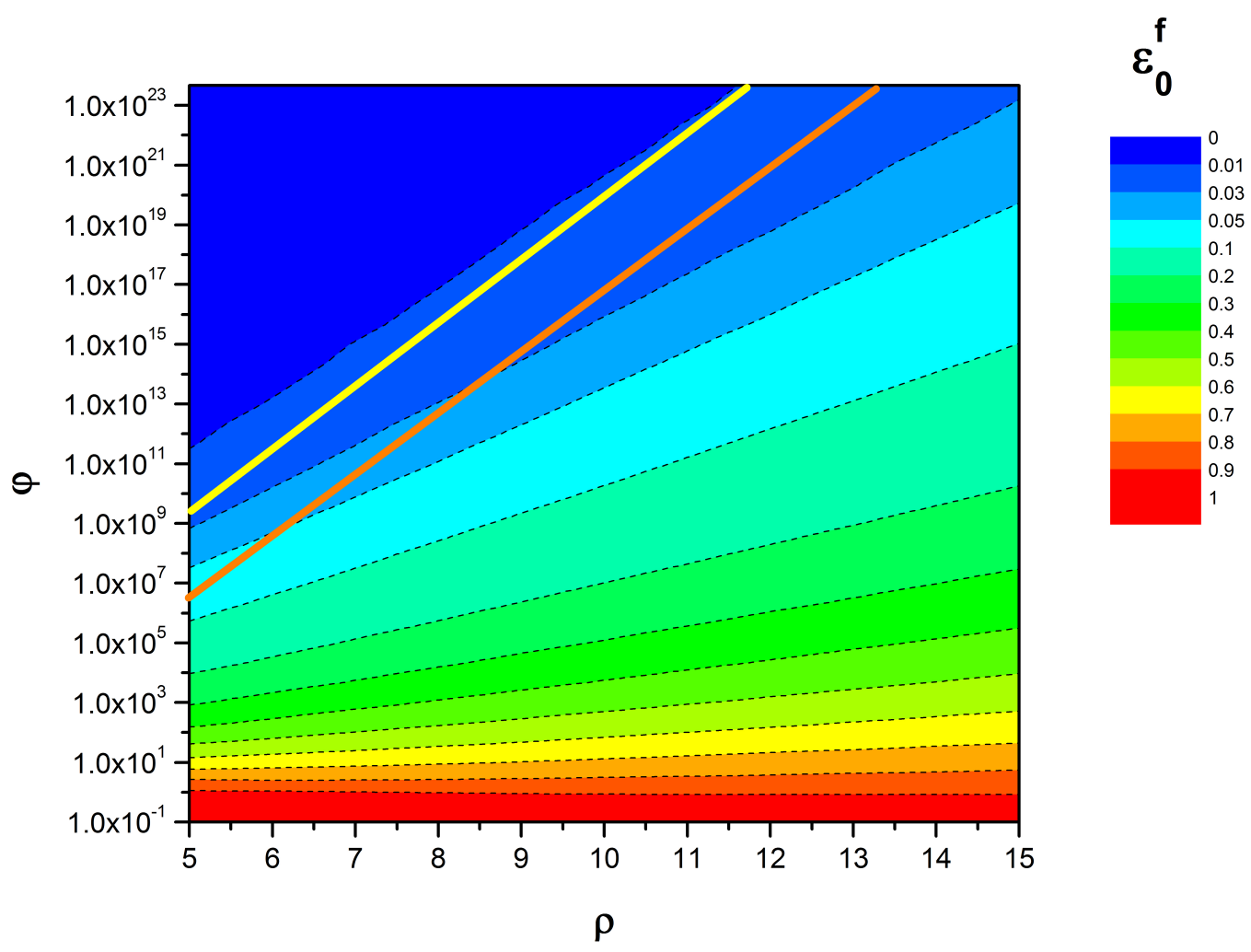

Figure 11: Maximum attainable strain in the uniform section $\left(\boldsymbol{\varepsilon}_{\mathbf{0}}^{\boldsymbol{f}}\right)$ of a composite combining a brittle phase with a ductile phase, as a function of the brittle phase dimensionless parameter $\varphi$ (Eq. (18)) and the Weibull modulus $\rho$, setting $\gamma=0$, but having $\mathrm{n}=0.3$ and $\mathrm{m}=0$, and for $\eta=0.005$. Yellow line: predicted ductility for a mediumstrength steel / aluminium alloy 6061 composite with $h=50 \mu \mathrm{m}$; Orange line: same but with $h=50 \mathrm{~nm}$. 
Table 2: Physical properties of brittle metals found in literature: $E, \sigma_{0}, \rho$ and $\mathrm{L}_{0}$, characterizing a two-parameter Weibull model. For different testing temperatures.

\begin{tabular}{|c|c|c|c|c|c|c|}
\hline Brittle metals & $\mathbf{E}[\mathbf{G P a}]$ & $\boldsymbol{\sigma}_{\mathbf{0}}[\mathbf{M P a}]$ & $\boldsymbol{\rho}$ & $\mathbf{T}\left[{ }^{\circ} \mathbf{C}\right]$ & $\mathbf{L}_{\mathbf{0}}[\mathbf{m m}]$ & Ref. \\
\hline A533B Steel & 215 & 11,000 & 4 & -150 & NA & {$[30]$} \\
\hline HT780 Steel & 206 & 2,690 & 12.2 & -75 & 1 & {$[29]$} \\
\hline A36 Steel & 200 & 1,800 & 7.8 & -43 & 1 & {$[28]$} \\
\hline Sand Cast A319 Aluminium & 74 & 197 & 21 & RT & NA & {$[31][32]$} \\
\hline Ductile cast Iron <4\% Pearlite & 170 & 73.5 & 15.7 & -40 & 1 & {$[33]$} \\
\hline Ductile cast Iron <9\% Pearlite & 170 & 71.4 & 11.7 & -40 & 1 & {$[33]$} \\
\hline Ductile cast Iron <20\% Pearlite & 170 & 69.4 & 9.47 & -40 & 1 & {$[33]$} \\
\hline SA508 Carbon Steel & 210 & 1,350 & 9 & -40 & NA & {$[58]$} \\
\hline $\begin{array}{c}\text { Al356 Vertically top filled Cast } \\
\text { Al-7Si-Mg }\end{array}$ & 71 & 289 & 10.8 & RT & NA & {$[34][35]$} \\
\hline
\end{tabular}

Summing up, what transpires most strongly from the present analysis is that an equistrain composite combining a brittle (two parameter Weibull) with a ductile (strain and strain-rate hardening) phase will nearly always have a rather low ductility: cracking in the brittle phase destabilizes, in nearly all practically relevant cases, tensile deformation of the composite, regardless of ductile phase properties. The main exception to this is in composites having very thin layer thicknesses: these can be made reasonably ductile because very short shear-lag lengths, coupled with strengthening of the ductile phase, lead to efficient damage healing, allowing in turn elongations that can significantly exceed one percent - provided of course that there is no clustering of damage (or fracture by local load sharing, in the parlance of composite mechanics). Best is, however, to avoid damage altogether: here again reducing layer thicknesses is a potent strategy. In recent research, laminated austenitic/martensitic steel composites having exceptional combinations of strength and ductility were demonstrated: key ingredients to this achievement were a suppression of tunnel crack growth in the brittle martensitic steel at sufficiently small layer thicknesses in laminated metal composites having a sufficiently strong and tough interface [59-61].

\section{Conclusion}

Shear-lag analysis of the deformation of equistrain composites in the presence of stochastic cracking of one phase is adapted for strain and strain-rate hardening in the other, ductile, phase, and then implemented in the Hutchinson-Neale-Ghosh long wavelength model for the prediction of the composite uniform strain to failure as governed by neck formation and instability. It is found that strain and strain-rate hardening in the ductile phase can delay somewhat the onset of unstable deformation; however, the resulting increase in composite elongation is generally small. It is furthermore found that only two parameters influence strongly the predicted composite tensile ductility: these are the brittle phase Weibull modulus $\rho$ and the dimensionless parameter $\varphi$ that is defined in Eq. (18). Working the model with practical values of its governing parameters leads to conclude that the best strategy to produce ductile laminated composites when one of its phases is susceptible to cracking is to reduce the scale of the microstructure: greater ductility is then obtained as damage in thinner layers or fibres is then more efficiently healed or suppressed. 


\section{Acknowledgements}

This work was sponsored by the Collaborative Research Programme between the University of Tokyo and the Ecole Polytechnique Fédérale de Lausanne (EPFL) supported by the Toyota Motor Corporation. The authors wish to thank Professors Toshihiko Koseki, Junya Inoue and Shoichi Nambu of the University of Tokyo, for the many interactions and exchanges we have had in the course of this collaboration. We also wish to thank Professor William Curtin at EPFL for several stimulating and enlightening discussions on the analysis presented here.

\section{References}

[1] Hull D, Clyne TW. Introduction to Composite Materials, Second Edition. Cambridge, UK: Cambridge University Press; 1996.

[2] Chawla KK. Composite Materials, Science and Engineering - Third Edition. New-York: Spring Verlag; 2012.

[3] Embury JD. Annual Review of Materials Research 2010;40:213.

[4] Mortensen A, Llorca J. Annual Review of Materials Research 2010;40:243.

[5] Fuwa M, Bunsell AR, Harris B. Journal of Materials Science 1975;10:2062.

[6] Rossoll A, Otto C, Moser B, Weber L, Wanner A, Mortensen A. Scripta Materialia 2008;59:842.

[7] Rossoll A, Moser B, Mortensen A. Composites Part A: Applied Science and Manufacturing 2012;43:129.

[8] Hauert A, Rossoll A, Mortensen A. Journal of the Mechanics and Physics of Solids 2009;57:473.

[9] Kouzeli M, Weber L, San Marchi C, Mortensen A. 2001;49:3699.

[10] Brockenbrough JR, Zok FW. Acta Metallurgica 1995;43:11.

[11] Lesuer DR, Syn CK, Sherby OD, Wadsworth J, Lewandowski JJ, Hunt WH. International Materials Reviews 1996;41:169.

[12] Hutchinson JW, Neale KW. Acta Metallurgica 1977;25:839.

[13] Hutchinson JW, Obrecht H. Proceedings of the 4th International Conference on Fracture 1977;1:101.

[14] Ghosh AK. Acta Metallurgica 1977;25:1413.

[15] Çetin A, Bernardi C, Mortensen A. Acta Materialia 2012;60:2265.

[16] Curtin WA. Advances in Applied Mechanics 1999;36:163. 
[17] Leigh Phoenix S, Beyerlein I. Statistical Strength Theory for Fibrous Composite Materials, in: Comprehenseive Composite Materials. Elsevier; 2000.

[18] Hui C, Leigh Phoenix S, Ibnabdeljalil M, Smith RL. Journal of the Mechanics and Physics of Solids 1995;43:1551.

[19] Neumeister JM. Journal of the Mechanics and Physics of Solids 1993;41:1383.

[20] Kelly A, Macmillan NH. Strong Solids, 3rd Edition, Oxford Sci. Oxford, UK: Clarendon Press; 1986.

[21] Wachmtman JB. Mechanical Properties of Ceramics. New-York: J. Wiley \& Sons; n.d.

[22] Meyers MA, Chawla KK. Mechanical Behaviour of Materials, 2nd Edition. Cambridge: Cambridge University Press; 2009.

[23] Curtin WA. Journal of American Ceramic Society 1991;45:2837.

[24] Curtin WA. Journal of Materials Science 1991;26:5239.

[25] Tejedor R, Rodriguez-Baracaldo R, Benito JA, Caro J, Cabrera JM. Scripta Materialia 2008;59:631.

[26] Lin MR, Wagoner RH. Scripta Metallurgica 1986;20:143.

[27] Miles MP, Siles JL, Wagoner RH, Narasimhan K. Metallurgical Transactions A 1993;24A:1143.

[28] Gao X, Ruggieri C, Dodds RH. International Journal of Fracture 1999;92:175.

[29] Yamashita Y, Minami F. Journal of ASTM International 2013;5:1.

[30] Esposito L, Gentile D, Bonora N. Engineering Fracture Mechanics 2007;74:549.

[31] Tiryakioğlu M, Campbell J. Metallurgical and Materials Transactions A 2010;41:3121.

[32] Zahedi H, Emamy M, Razaghian a., Mahta M, Campbell J, Tiryakioğlu M. Metallurgical and Materials Transactions A 2007;38:659.

[33] Baer W, Wossidlo P, Abbasi B, Cassau M, Häcker R, Kossert R. Engineering Fracture Mechanics 2009;76:1024.

[34] Green N, Campbell J. Materials Science \& Engineering A 1993;173:261.

[35] Eisaabadi GB, Davami P, Kim SK, Tiryakio M. Materials Science \& Engineering A 2013;579:64.

[36] Cias a., Wronski a. S. Powder Metallurgy 2010;53:328.

[37] Klein CA. Optical Engineering 2011;50:1.

[38] Klein CA, Miller RP. Proceeding of SPIE 2001;4375:241. 
[39] Klein CA, Miller RP. Optical Engineering 2002;41:3151.

[40] Klein CA. Journal of Applied Physics 2007;101:1.

[41] Klein CA. Journal of Applied Physics 2008;104:1.

[42] Klein CA. Optical Engineering 2010;49:1.

[43] Xu ZR, Chawla KK, Li X. Materials Science \& Engineering A 1993;171:249.

[44] Ramamurty U. Composites Science and Technology 2005;65:1815.

[45] Hager IZ. Journal of Materials Science 2002;37:1309.

[46] Chang YS, Lee TR, Choi JB, Kim YJ, Kim MC, Lee BS. Key Engineering Materials 2006;326328:931.

[47] Knott JF. Structural Integrity and Fracture 2004:1.

[48] Lambrigger M. Journal of Materials Science 1999;34:4457.

[49] Gao H-L, Shen Y, Xu J. Journal of Materials Research 2011;26:2087.

[50] Lambrigger M. Journal of Materials Science 1999;34:4447.

[51] Curtin WA, Ahn BK, Takeda N. Acta Materialia 1998;46:3409.

[52] Ji S, Yang W, Jiang B, Patel JB, Fan Z. Materials Science and Engineering: A 2013;566:119.

[53] Beleznai R, Lenkey GB, Lauerova D. Journal of Nuclear Materials 2010;406:119.

[54] Gao X, Dodds RH. Engineering Fracture Mechanics 2005;72:2416.

[55] Niranjani VL, Hari Kumar KC, Subramanya Sarma V. Materials Science and Engineering: A 2009;515:169.

[56] Wadley HNG, Hsiung LM, Lankey RL. Composites Engineering 1995;5:935.

[57] Calhoun RB, Dunand DC. Dislocations in Metal Matrix Composites, Chapter 3.02, in: Comprehensive Composite Materials, T. W. Clyn. Oxford, UK: Pergamon Press; 2000.

[58] Chang YS, Lee TR, Choi JB, Kim YJ, Kim MC, Lee BS. Key Engineering Materials 2006;326328:931.

[59] Inoue J, Nambu S, Ishimoto Y, Koseki T. Scripta Materialia 2008;59:1055.

[60] Nambu S, Michiuchi M, Inoue J, Koseki T. Composites Science and Technology 2009;69:1936.

[61] Koseki T, Inoue J, Nambu S. Materials Transactions of the Japan Institute of Metals and Materials 2014:in print. 
